\title{
Simultaneous Full-Field Strain and Temperature Measurements in Tensile Hopkinson Bar Experiments at Extreme Temperatures
}

\author{
Guilherme C. Soares* and Mikko Hokka \\ Impact - Multiscale Mechanics Research Group, Engineering Materials Science, Tampere University, \\ POB 589, FI-33014, Tampere, Finland
}

\begin{abstract}
Simultaneous full-field strain and temperature measurements were used to monitor tension Split Hopkinson Pressure Bar (SHPB) tests at elevated temperatures. A direct heating system was used to increase the specimen temperature up to $1350{ }^{\circ} \mathrm{C}$. Digital Image Correlation (DIC) and Infrared Thermography (IRT) were used was used to simultaneously monitor the evolution of the full-field strain and temperature of the specimen. Data acquisition was synchronized using a function generator, a camera pinhole model was used to represent both strain and temperature on the same coordinate system, and the displacement vector field from DIC was used to represent both datasets on the same reference frame. The use of fullfield techniques was essential at elevated temperatures, as necking occurred soon after yielding and the usability of the data obtained from the SHPB after the onset of necking is debatable. The method was able to follow the full-field strain and the temperature evolution under extreme conditions. Some challenges were found in the development of the method and recommendations as well as future applications are also described in this paper. This experimental approach is versatile and can be applied to different materials at similar testing conditions but also different loading modes and testing setups.
\end{abstract}

\section{Introduction}

The design and modelling of material behaviour in certain applications, such as airplanes and spacecrafts, must consider the mechanical behaviour of materials at high temperatures and under dynamic loading events [1]. The use of full-field techniques such as Digital Image Correlation (DIC) and Infrared Thermography (IRT) have become increasingly popular in characterization of dynamic material behaviour. These techniques are powerful and versatile, allowing researchers to investigate complex material behaviour. DIC enables measurements of localized strains and IRT allows the measurements of local temperatures during mechanical loading, and therefore a much more comprehensive description of the thermomechanical response of the material to dynamic loading can be described with high spatial resolution. Although there already are works that use DIC and IRT to investigate

*Corresponding author: guilherme.correasoares@tuni.fi 
material behaviour at high strain rates $[2,3]$, full-field strain and temperature measurements at elevated temperatures have only been possible at lower acquisition rates [4-6]. The use of DIC and IRT for measuring the full-field strains and temperatures at high strain rates and elevated temperatures has not yet been reported on the literature.

This work presents an application of synchronized full-field strain and temperature measurements to investigate tensile response of materials under high strain rates and elevated temperatures. Some of the challenges and solutions involved in the measurements are described with examples. Suggestions are also given on how to concisely present such fullfield data sets and facilitate the comparison between different materials and tests.

\section{Experimental Procedure}

A commercially pure $\alpha$-titanium alloy was used in this investigation, and special dog-bone tensile samples were machined from a $2 \mathrm{~mm}$ thick sheet using electric discharge machining. The tensile tests were conducted with a tensile Split Hopkinson Pressure Bar (SHPB) device with a direct electric heating system, which enables rapid heating of the sample to the test temperature. The tensile SHPB comprised a steel incident bar, an aluminium bar, and a tubular striker which is accelerated using compressed air. The stress pulses in the incident and transmitted bars were measured by two $5 \mathrm{~mm}$ strain gauges, amplified by a signal conditioner, and recorded on a digital oscilloscope. A complete description of this setup and examples of its use can be found in references [7,8].

The heating system comprises a welding transformer and pneumatic actuators which grip the sample. The welding transformer passes a current through the gauge section of the specimen and the pneumatic actuators disengage the specimen just moments before the loading pulse reaches the specimen. Due to the short time that the specimen stays at the elevated temperatures, the heat diffusion to the bars is greatly diminished, the oxidation of specimen surface is decreased, and the applied contrast patterns remain ductile on the specimen surface for a longer time and can follow the deformation of the tensile specimen. More detailed information on the heating system can be found in references [1,9]. The tests were conducted at strain rates from 1000 to $1600 \mathrm{~s}^{-1}$ and temperatures from room temperature up to $1350{ }^{\circ} \mathrm{C}$.

The tests were monitored by two Photron SA-X2 high-speed cameras and a Telops FASTIR-M2K high speed infrared camera. All cameras acquired images at a rate of $62 \mathrm{kHz}$. The optical cameras imaged one side of the specimen with a random pattern prepared on the surface, while the infrared camera imaged the opposite side of the specimen where the surface was only ground and clean. Cold LED lights were used to illuminate the specimen for the optical imaging.

The radiometric temperature values from the infrared camera consider an emissivity of a perfect black body, or a constant determined by the user, and thus had to be converted to true surface temperatures. This calibration was done by monitoring the cooling down of hot specimens simultaneously with a spot welded type K thermocouple and the infrared camera. The strain and temperature on both sides of the specimen were considered to be the same, as the specimens were thin metal sheets. A Keysight 33500B waveform generator and an analog gate were used to synchronize the data acquisition of all measurement systems. The waveform generator sends TTL pulses that clock the optical cameras and triggered the image acquisition of the infrared camera. The analog gate prevents the TTL pulses from reaching the infrared camera until the gate is opened by the oscilloscope trigger out signal. A general view of the experimental setup is shown in Figure 1. 


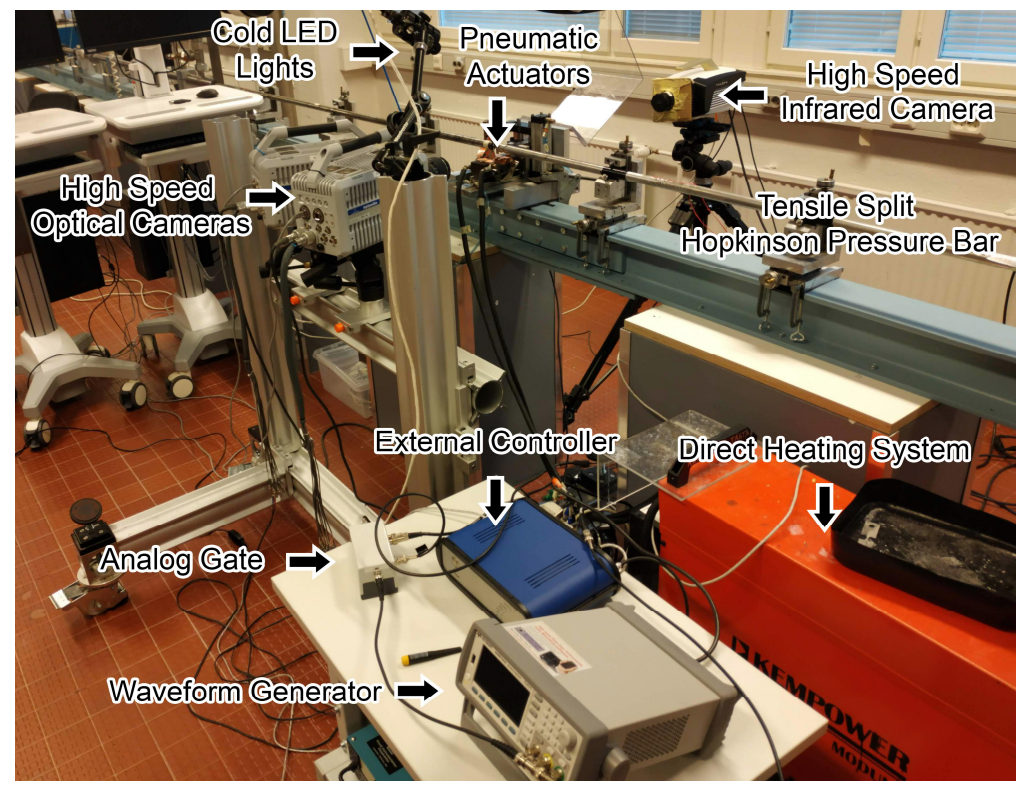

Fig. 1. Experimental setup used for the high temperature tests comprising the direct electric heating system, the high-speed infrared, and optical cameras, and the tensile SHPB.

A random speckle pattern was applied to one side of the sample using a temperature resistant manifold spray paint which resisted temperatures up to $1120{ }^{\circ} \mathrm{C}$. Despite most of the pattern evaporated at the temperature of $1350^{\circ} \mathrm{C}$, DIC measurements were still possible using the remnants of the spray-painted pattern and the surface texture caused by plastic deformation. A bandpass filter $(410-570 \mathrm{~nm})$ was used with the optical cameras to filter most of the incandescent radiation emitted by the specimen at elevated temperatures. An attenuation filter had to be used with infrared camera so that imaging of a higher temperature range would be possible. The infrared camera must be refocused after the attenuation filter is applied and this was done by pursuing sharp edges while imaging a hot soldering iron.

A two-level double-sided 3D calibration plate was used to calibrate all three cameras to a single pinhole model, making it possible to overlap the images from both optical and infrared cameras by representing both datasets in the same world coordinate system. Considering that the attenuation filter changes the optics of the infrared camera, the infrared images of the calibration plate had to be taken while having the filter equipped. The calibration plate could not be heated up so that it would emit enough radiation to be imaged by the infrared camera, and therefore an approach of using reflected infrared radiation from the surface of the calibration plate was used. Non-Uniformity Correction (NUC) maps were used to acquire these images, as they allowed for better intensity imaging of the calibration plate through the attenuation filter. Adequate results were obtained by using either a ringshaped heater or an infrared bulb, but the former was chosen as it produced less shadows.

The IRT images were deformed using the displacement vector fields obtained from DIC so that both temperature and strain data could be analysed in the reference frame of the undeformed sample. A more detailed description of the temporal synchronization, spatial calibration, and representation on the data in the same frame of reference can be found in reference [2]. At elevated temperatures, the specimens did not always break during the first loading pulse. However, it was possible to obtain full-field information from the sample during the second loading pulse as well and measure the full-field strain/temperatures up to the rupture of the specimen. These results were represented as full-field maps overlayed on top of the optical images and as 3D waterfall plots which allowed for an easier comparison 
of the results at different testing conditions. The noise-floor of the strain and displacement measurements of DIC doubled at higher temperatures but stayed within an acceptable range.

\section{Results}

The mechanical strength of the material decreased drastically at elevated temperatures while the ductility increased, mostly due to a much higher non-uniform deformation. At higher temperatures, necking was very pronounced and started at the very beginning of plastic deformation. The strain localization at elevated temperatures is much higher than that at room temperature. The evolution of the full-field strains and temperatures as a function of engineering strain for a sample tested at an average temperature of $1350^{\circ} \mathrm{C}$ and a strain rate of $1600 \mathrm{~s}^{-1}$ is shown in Figure 2. Even though most of the contrast pattern evaporated at this temperature, the reimaging pattern and the surface features allowed for strain measurements to be made. Axial strain mostly concentrated on the hotter parts of the specimen and no discernible adiabatic heating was observed under these conditions.

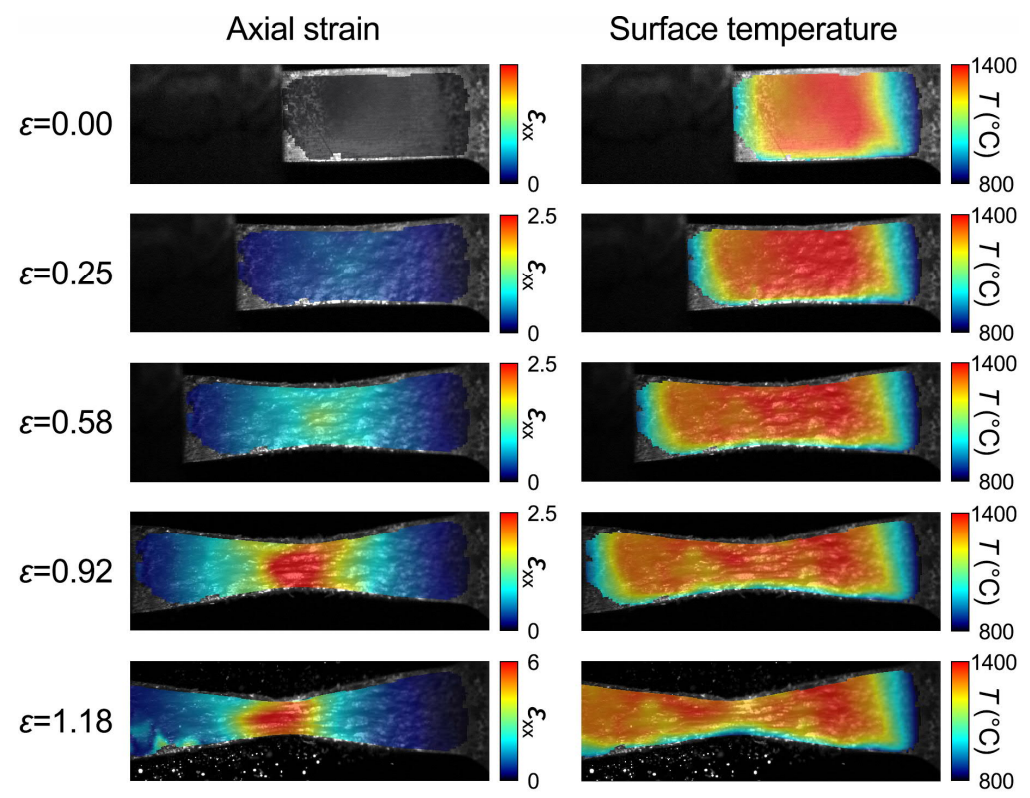

Fig. 2: Full-field axial strain and temperature maps overlayed on the optical images of the specimen at different overall engineering strains of a commercially pure titanium specimen tested in tension at a strain rate of $1600 \mathrm{~s}^{-1}$ and an initial temperature of approximately $1350{ }^{\circ} \mathrm{C}$.

The full-field data from the same test at $1350{ }^{\circ} \mathrm{C}$ is further presented in Figure 3 as 3D waterfall plots where strain and temperature are shown as a function of position along the gauge length of the specimen and global engineering strain. The highest measured local axial strain was of almost 10. The specimen had a temperature gradient throughout its gauge length prior to testing, and while the average temperature did not change during the test the temperature data obtained in some regions was less stable during the experiment at these extreme conditions. This could possibly be caused local changes in the emissivity of the surface caused by the plastic deformation and oxidation. There was no noticeable relationship between the strain and temperature evolution in the test at $1350{ }^{\circ} \mathrm{C}$. The darker line in the plot at an engineering strain of approximately 1, originates from the data obtained from the pictures that were taken in between loading pulses during which the specimen was not loaded. It is also evident from this plot that there is no considerable decrease in temperature between 
the loading pulses. Presenting the full-field data as 3D waterfall plots allow for data from all acquired images to be observed at a quick glance and makes it easier to compare the strain localization and adiabatic heating among different testing conditions and materials.


Fig. 3: 3D waterfall plots of axial strain and temperature as a function of position along the gauge length of the specimen and global engineering strain of a commercially pure titanium specimen tested in tension at a strain rate of $1600 \mathrm{~s}^{-1}$ and an initial temperature of approximately $1350{ }^{\circ} \mathrm{C}$.

Another compelling form of presenting such full-field data in an even more compact manner is a surface plot where axial strain is shown as a function of position along the gauge length and global engineering strain, and where the local temperature is represented by the colour. Figure 4 shows an example of such a plot which illustrates the strain and temperature evolution of a specimen tested at an initial temperature of $700{ }^{\circ} \mathrm{C}$ and a strain rate of 1600 $\mathrm{s}^{-1}$. This plot shows well that both the full-field strain and temperature data are temporally and spatially synchronized, and properly presented on the same frame of reference. Although this test was carried out at elevated starting temperature, considerable adiabatic heating was observed first throughout the gauge length of the specimen and later concentrated on the necking region only. Increasing temperature was observed up to an axial strain of 2, but the adiabatic heating slowed down significantly even when the localization became more evident. Strain localization at $700{ }^{\circ} \mathrm{C}$ is smaller than at $1350{ }^{\circ} \mathrm{C}$ and the maximum local axial strain were of approximately 4.5 .

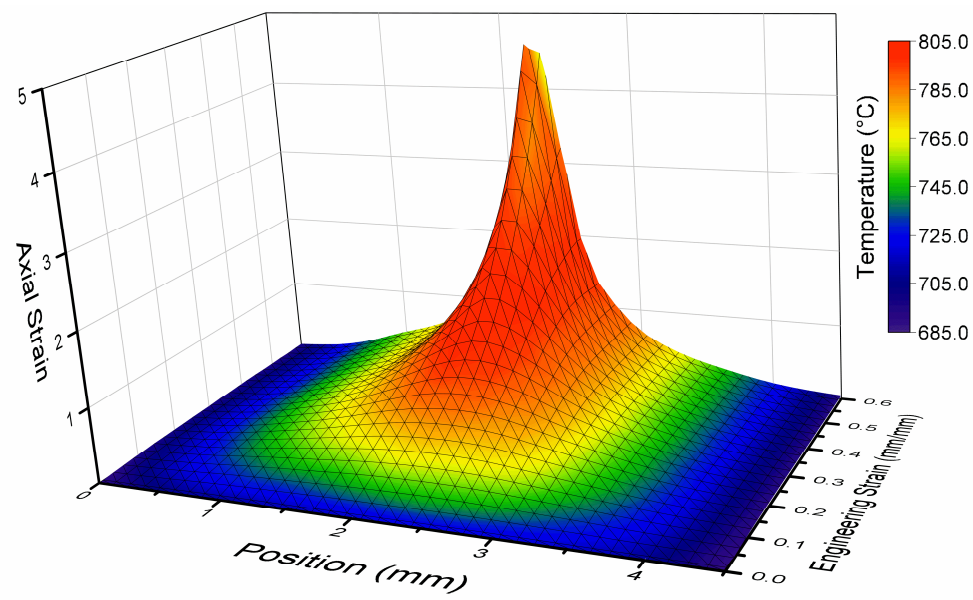

Fig 4. Surface plot of local axial strain as a function or position along the gauge length and global engineering strain of a specimen under tension at a strain rate of approximately $1600 \mathrm{~s}^{-1}$ and an initial temperature of $700{ }^{\circ} \mathrm{C}$. The plot is colour coded so that it possible to visualize the local surface temperature of the specimen in the same representation as the local axial strain. 


\section{Summary}

This work presented the use of simultaneous full-field strain and temperature measurements to monitor high strain rate tension tests at temperatures up to $1350^{\circ} \mathrm{C}$. Several practical complications arose during the development of this method and most of them were successfully dealt with. For example, incandescence was solved by bandpass filters, and a resistance heating ring was used to enable infrared imaging of the calibration plate with attenuation filters on the infrared camera. Nevertheless, there are still many challenges that need to be addressed to enable the testing at even higher temperatures.

The mechanical resistance of commercially pure titanium decreased considerably, and ductility increased greatly at elevated temperatures. The full-field techniques were essential in obtaining data from the tests at elevated temperatures, considering that necking occurred shortly after yielding in the investigated material and most of the test consisted of nonuniform deformation. Additionally, even though specimens above a certain temperature did not break on the first loading pulse, this method also allowed for the full-field strain and temperature to be monitored during the second loading pulse up until fracture. Most of the data acquired from these tests would not have been accessible with only traditional testing methods.

This data can be useful for improving the current understanding of the thermomechanical behaviour material under extreme condition and improving material modelling and simulation of applications under such conditions. This experimental approach is very versatile and can be applied to different materials at similar testing conditions but also different loading modes and testing setups at high temperature.

This work was financially supported by the Air Force Office of Scientific Research (AFOSR).

\section{References}

1. F. Hueto, M. Hokka, R. Sancho, J. Rämö, K. Östman, F. Gálvez, V.-T. Kuokkala, Procedia. Engineer. 197, 130 (2017)

2. G. C. Soares, N. I. Vázquez-Fernández, M. Hokka, Exp. Tech. (2021) DOI: 10.1007/s40799-020-00436-y

3. J. D. Seidt, V.-T. Kuokkala, J. L. Smith, A. Gilat. Exp. Mech. 57, 219 (2017)

4. Y. Tang, M. Yue, X. Fang, X. Feng, Opt. Laser. Eng. 116, 94 (2019)

5. Y. Tang, J. Zhang, M. Yue, X. Zhu, X. Fang, X. Feng, Opt. Laser. Eng. 137, 106361 (2021)

6. J. J. Orteau, Y. Rotrou, T. Sentenac, L. Robert, Exp. Mech. 48, 163 (2008)

7. M. Hokka, Effects of Strain Rate and Temperature on the Mechanical Behavior of Advanced High Strength Steels, Doctoral Thesis, Tampere University of Technology (2008)

8. S. Curtze, Characterization of the dynamic behavior and microstructure evolution of high strength sheet steels, Doctoral Thesis, Tampere University of Technology (2009)

9. M. Hokka, K. Östman, V.T. Kuokkala, High Temperature Tension HSB Device Based on Direct Electrical Heating, in Dynamic Behavior of Materials, Volume 1. Conference Proceedings of the Society for Experimental Mechanics Series (2015) DOI: 10.1007/978-3-319-06995-1_34 